\title{
In vivo SELEX of bone targeting aptamer in prostate cancer bone metastasis model
}

\author{
Lingxiao Chen ${ }^{1, *}$ \\ Wei He ${ }^{1, *}$ \\ Huichuan Jiangl,* \\ Longxiang $\mathrm{Wu}^{\prime}$ \\ Wei Xiong' \\ Bolun $\mathrm{Li}^{\prime}$ \\ Zhihua Zhou ${ }^{2}$ \\ Yuna Qian ${ }^{3-5}$ \\ 'Department of Urology, Xiangya \\ Hospital, Central South University, \\ Changsha, Hunan 4l0008, China; \\ ${ }^{2} \mathrm{School}$ of Chemistry and Chemical \\ Engineering, Hunan University of \\ Science and Technology, Xiangtan, \\ Hunan 4I I 20I, China; ${ }^{3}$ Wenzhou \\ Institute of Biomaterials and \\ Engineering, Chinese Academy of \\ Science, Wenzhou, Zhejiang 32500 I, \\ China; ${ }^{4}$ School of Ophthalmology and \\ Optometry, School of Biomedical \\ Engineering, Wenzhou Medical \\ University, Wenzhou, Zhejiang \\ 325035, China; ${ }^{5}$ Key Laboratory of \\ Biorheological Science and Technology \\ (Chongqing University), Ministry of \\ Education, Chongqing 400045, China \\ *These authors contributed equally \\ to this work
}

This article was published in the following Dove Medical Press journal: International Journal of Nanomedicine
Purpose: PB is one of the most severe complications of late stage prostate cancer and negatively impacts patient quality of life. A major challenge for the treatment of cancer bone metastasis is the management of efficient drug delivery to metastatic bone lesion. We aimed to explore the use of aptamers as promising tools to develop a targeted drug delivery system for PBs.

Materials and methods: In vivo SELEX was applied to identify bone targeting aptamer in a mouse model with PBs.

Results: The aptamer (designated as "PB") with the highest bone targeting frequency in mice bearing PC3 PB was selected for further analysis. The PB aptamer specifically targeted modulated endothelial cells in response to cancer cells in the bones of mice bearing PC3 PBs. The targeting efficiency of the PB aptamer conjugated to gold particles was verified in vivo.

Conclusion: This investigation highlights the promise of in vivo SELEX for the discovery of bone targeting aptamers for use in drug delivery.

Keywords: aptamer, tumor endothelial targeting, drug delivery

\section{Introduction}

Prostate cancer is the most common noncutaneous cancer in men in the USA. In 2017, 160,000 men were diagnosed with prostate cancer, adding to 3.3 million existing survivors. ${ }^{1}$ Most cancer-related deaths are caused by metastases in vital organs, ${ }^{2}$ with bones among the most frequent sites of prostate cancer metastases. Therefore, the identification of effective treatments for bone metastases is an ongoing important topic in clinical practice related to prostate cancer.

Current treatments for PB are limited. Typically, radiation is used to inhibit tumor growth within bone; however, it is insufficiently effective. Bisphosphonates and denosumab are the two major clinical drugs used for palliative treatment to avoid bone-related events. ${ }^{3,4}$ Although such palliative treatment drugs delay cancer progression by inhibiting osteolysis in metastatic prostate cancer, they are not effective in killing cancer cells and do not confer patient any survival benefit. To survive in the bone marrow, cancer cells modulate the local environment to support tumor growth by stimulating stromal cells to secrete copious tumor growth factors, which accelerate tumor progression. ${ }^{5-7}$ The eradication of cancer cells in bone metastases is crucial for the treatment of $\mathrm{PB}$, and precise and efficient delivery of cancer therapeutics to bone is the essential issue.

To resolve this problem, different synthetic carriers, based on cationic polymers, peptides, and lipids, have been developed to form compact nano-sized complexes carrying cancer therapeutics to specific tumor lesion. ${ }^{8}$ However, the side effects 
caused by off-target effects have resulted in low therapeutic index and poor safety profiles, which cannot be ignored. ${ }^{9}$ To improve the therapeutic index and safety of potential therapeutics, one promising approach is to transport drugs to their specific target destinations and shield the drugs from elimination and degradation in the process of overcoming biological barriers. ${ }^{10}$

Aptamer is a specialized type of small single-stranded RNA or DNA nucleic acid. In a manner analogous to the function of antibodies, the particular spatial structure of aptamer can identify and bind to unique ligand ranging from a small organic molecule to a whole cell with high specificity and affinity. The unique properties of nucleotide acids provide aptamers with numerous advantages relative to protein antibodies, including fast tissue penetration, nonimmunogenicity, and thermal stability. ${ }^{11}$ More significantly, the convenience ability of nucleotides in conjugation with diverse functional moieties and the extensive scope of their potential biological ligands make aptamer a promising targeting tool in the development of targeted delivery system.

In this research, we applied in vivo SELEX to identify bone targeting aptamer, with the aim of achieving targeted drug delivery using a nude mouse model bearing PC3 PBs. A thioaptamer library was intravenously injected into nude mouse with PC3 PBs, and residual bone marrow aptamers were amplified for subsequent rounds of SELEX. After ten rounds, the aptamer with the highest hitting point (referred as the "PB" aptamer) for bone in the diseased mice was obtained and further characterized in vitro and in vivo. The PB aptamer could not only exhibit high levels of accumulation in the bone of diseased mice through targeting endothelial cells but also specifically target bone in diseased mice, based on recognition of endothelial cells modulated by the local tumor microenvironment. Lastly, the PB aptamer-conjugated gold nanoparticle as targeted delivery system turned out to be of high efficiency in bone accumulation in mice with PBs in a local endothelial cell targeting manner, which validated the original purpose of our study.

\section{Materials and methods Cell culture}

The luciferase-labeled PC3 prostate cancer cell line, DU145 cells, and LNCaP cells were from Nanjing COBIOER BIOSCIENCES. PC3, DU145, and LNCaP cells were cultured in RPMI-1640 (Invitrogen) with 10\% FBS (Invitrogen) and 1\% penicillin-streptomycin (Invitrogen). Human umbilical vein endothelial cell (HUVEC) was also from Nanjing COBIOER BIOSCIENCES and was cultured in M199 (HyClone) added with 15\% FBS (Invitrogen), 1\% endothelial cell growth supplement, and heparin. Cells were incubated at $37^{\circ} \mathrm{C}, 5 \%$ $\mathrm{CO}_{2}$, and $100 \%$ humidity.

\section{Murine model of human PB}

The use of animals was conformed and approved by the Guiding Principles in the Care and Use of Animals for Xiangya Hospital. Animal work was processed following animal experiment regulation of Xiangya Hospital. Sixweek-old male athymic nude mice were purchased from the Shanghai SLAC Laboratory Animal. They were housed in a pathogen-free facility and fed with a pathogen-free diet and water. To build up murine models bearing prostate cancer bone metastasis, $1 \times 10^{5}$ luciferase-labeled PC3 cells were inoculated into mice by intracardiac inoculation. Bone metastasis development was observed under a Xenogen IVIS 200 imaging system. Mice with extensive tumor signal (at least one strong luminescence signal over 60,000 in both spine and limbs) in bone were picked for experiments.

\section{Thioaptamer library}

A library of random ssDNA oligonucleotides was from BD Biosciences. This library consisted of a 30 base random region with a flanker of a 21 base $5^{\prime}$-primer $\left(5^{\prime}\right.$-CGCTCGATCGATCTAGATTCG-3') and a 21 base $3^{\prime}$-primer (5'-GTCATCACGCTCAGATCACTG-3'). PCR amplification was repeated using the extracted ssDNA oligonucleotides after each selection round. First, the ssDNA random library was incubated with Taq DNA polymerase in a mixture of dATP, dTTP, dCTP, and dGTP (500 $\mu \mathrm{M}$ each) for 5 hours at $37^{\circ} \mathrm{C}$. After reverse strand was synthesized, the dsDNA library (400 nM) went through PCR amplification. Then PCR products were filtered by a Millipore YM-30 filter to purify dsDNA product. The product was incubated with streptavidin-coated magnetic beads in buffer $(10 \mathrm{mM}$ Tris-HCl, $2 \mathrm{M} \mathrm{NaCl}$, and $1 \mathrm{mM}$ EDTA [pH 7.5]) for 20 minutes. Biotin on the reverse strand of the dsDNA binds to the streptavidin beads, followed by separation of the two DNA strands in melting solution $(0.1 \mathrm{M} \mathrm{NaOH})$. The forward strands were collected after filter with Millipore YM-10 filter for the next round of selection.

\section{In vivo SELEX}

Nude mouse bearing PC3 PBs was chosen to perform aptamer SELEX by a library composed of different ssDNA 
sequences. When the tumor models were ready about 4-6 weeks after inoculation (nude mouse at the middle stage of PC3 PB development before body paralysis), $10 \mu \mathrm{g}$ thioaptamer library was intravenously injected to mice. After 4 hours circulation, mice were sacrificed, and bone marrow was collected and frozen in liquid nitrogen. Tissues were homogenized, and DNA was extracted for PCR amplification with aptamer library-specific primers. PCR products were recovered and purified for next round of screening. After ten rounds of screening, high-throughput sequencing was applied, and high-frequency sequences were validated. Nonrelevant aptamer (5'-CTACTGGACTTCATCGGAGCTAGGTCATCGCTTGCATGCA-3') was chosen from reference paper as a negative control. ${ }^{12}$

\section{Accumulation of PB aptamer in bone}

Biophotonic imaging technology was used to examine aptamer distribution. To test bone targeting efficiency of $\mathrm{PB}$ aptamer, $0.5 \mathrm{nmol} \mathrm{Cy} 5-\mathrm{PB}$ or $\mathrm{Cy} 5-\mathrm{Control}$ aptamer was injected in nude mice with $\mathrm{PC} 3 \mathrm{PBs}(n=6)$. Four hours after injection, mice were sacrificed and $\mathrm{Cy} 5$ signal intensity of major skeletal organs (spine, femur, and tibia) was measured by IVIS-200 system ex vivo. To test whether PB aptamer has targeting specificity for bone with tumor, $0.5 \mathrm{nmol} \mathrm{PB}$ aptamer was also injected in healthy nude mice as a contrast.

\section{PB aptamer bone targeting characterization}

To see the biodistribution of PB aptamer in vivo, Cy5-PB aptamer-injected nude mouse bearing PC3 PB were sacrificed and major skeletal organs were gathered. After fixation in $10 \%$ formalin, decalcification in 10\% EDTA, and embedding in paraffin, the skeletal organs were cut into $4 \mu \mathrm{m}$ sections, which were processed with $\mathrm{H} \& \mathrm{E}$ staining or stained with rat anti-mouse CD31 antibody (1:100 diluted, BD Biosciences) and second Alexa Fluor ${ }^{\circledR} 488$ donkey anti-rat antibody (1:100 diluted, Life Technologies). Sections were observed under a Nikon Eclipse 80i microscope.

To test whether PB aptamer can bind with bone endothelial cell of nude mice bearing PC3 PB, tumor mouse was sacrificed and major skeletal organs (spine, femur, and tibia) were gathered and smashed. Followed by digestion in DMEM with $1 \mathrm{mg} / \mathrm{mL}$ collagenase III for 1 hour, single cells were collected and incubated with PB aptamer or control aptamer $(25,50$, and $100 \mathrm{nM})$ in aptamer binding buffer $(4.5 \mathrm{mg} / \mathrm{mL}$ glucose, $1 \mathrm{mg} / \mathrm{mL}$ BSA, $0.1 \mathrm{mg} / \mathrm{mL}$ tRNA, $5 \mathrm{nM} \mathrm{MgSO} 4)$ on ice for 0.5 hour. Cells were washed by washing buffer, and cells were suspended in washing buffer for flow cytometry analysis and aptamer binding with endothelial cell was measured by flow cytometry.

To test binding affinity of PB aptamer for HUVEC grown in different conditioned medium, medium conditioned with bone marrow was prepared by culturing healthy nude mouse bone marrow with RPMI-1960 for 2 days followed by recollection of the cultured medium. Similarly, medium conditioned with PC3 prostate cancer cell and bone marrow containing PC3 prostate cancer cells were also prepared. Then, HUVEC was cultured with primary RPMI-1960, bone marrow-conditioned RPMI-1960, PC3 prostate cancer cell-conditioned RPMI-1960, or bone marrow with prostate cancer cell-conditioned RPMI-1960 for 8 hours, respectively. After that, $\mathrm{PB}$ aptamer binding affinity with HUVEC grown in different medium was tested. PB aptamer uptake in HUVEC grown in conditioned medium was also visualized under fluorescence microscopy briefly. HUVEC grown in conditioned medium was fed with $50 \mathrm{nM}$ Cy5-PB aptamer for 0.5 hour, and then stained with Alexa Fluor 488 Phalloidin (1:100 diluted, Thermo Fisher Scientific) and DAPI ( $1 \mu \mathrm{g} / \mathrm{mL}$, Thermo Fisher Scientific). Effect of bone marrow containing LNCaP or DU145 prostate cancer cells conditioned medium on PB aptamer uptake in HUVEC was also measured.

\section{Cellular internalization pathways of PB aptamer}

To identify cellular internalization pathways of PB aptamer, uptake of PB aptamer by HUVEC at the presence of internalization pathway inhibitors was tested. The internalization pathway inhibitors include cytochalasin D $(80 \mu \mathrm{M}, \mathrm{Abcam})$, amiloride (400 $\mu \mathrm{M}$, Sigma-Aldrich Co.), chlorpromazine (50 $\mu \mathrm{M}$, MP Biomedicals), genistein (80 $\mu \mathrm{M}$, SigmaAldrich Co.), and dynasore (400 $\mu \mathrm{M}$, Abcam). HUVECs were preincubated with internalization pathway inhibitors for 30 minutes and then $25 \mathrm{nM} \mathrm{Cy5-PB}$ aptamer was added. After 30 minutes incubation, live cells were collected for cytometry analysis.

\section{PB aptamer tracking in HUVEC}

To test whether PB aptamer can escape from lysosome after cellular internalization, HUVEC was cultured in chamber slides and incubated with $100 \mathrm{nM}$ PB aptamer for 1 hour, 
4 hours, and 6 hours, respectively. Lysotracker (1:100 diluted, Cell Signaling) was added 0.5 hour prior to the end. After the incubation, medium was removed and cells were washed by PBS, followed by nuclear staining with DAPI. Cells were observed under a Nikon Eclipse 80i microscope.

\section{PB aptamer-conjugated gold particle bone targeting}

To test whether PB aptamer can be used as a bone targeting moiety for bone-targeted drug delivery, Cy5-PB thioaptamer was conjugated onto gold particles with a diameter of $1 \mu \mathrm{m}$ by modifying gold particle with 3-aminopropyltriethoxysilane and using 1-ethyl-3-(3-dimethylaminopropyl) carbodiimide hydrochloride as a poly linker for conjugation of PB aptamer. One billion $\mathrm{Cy} 5-\mathrm{PB}-\mathrm{Au}, \mathrm{Cy} 5-\mathrm{Au}$ particles were injected into mouse bearing PB ( $n=6)$. After 4 hours, mice were sacrificed and major skeletal organs were collected. Tissues were processed as described above. Gold particles with bright fluorescence were counted under Nikon Eclipse 80i microscope at 40× magnitude, and average particle numbers were counted from ten views of each sample.

\section{Statistical analysis}

For statistical comparisons, Student's $t$-test was applied (twotailed distribution, two-sample equal variance) in two-group comparison. In three-group comparison, ANOVA test was performed. Data were presented as mean $\pm \mathrm{SD}$. A value of $P<0.05$ was considered statistically significant.

\section{Results}

\section{Selection of the aptamer most strongly targeting bone}

After ten continuous rounds of in vivo SELEX in nude mouse bearing PC3 PB (Figure 1A and B), three aptamer candidates with the strongest bone marrow targeting capability were identified. The aptamer with the highest hit frequency (sequence: 5'-CTCTATTGATGCCTGCGTGCGTGCTTG-
A

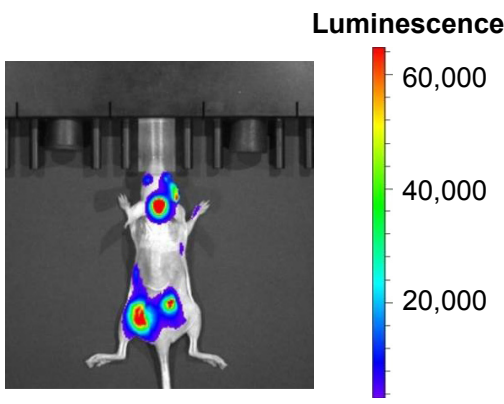

B

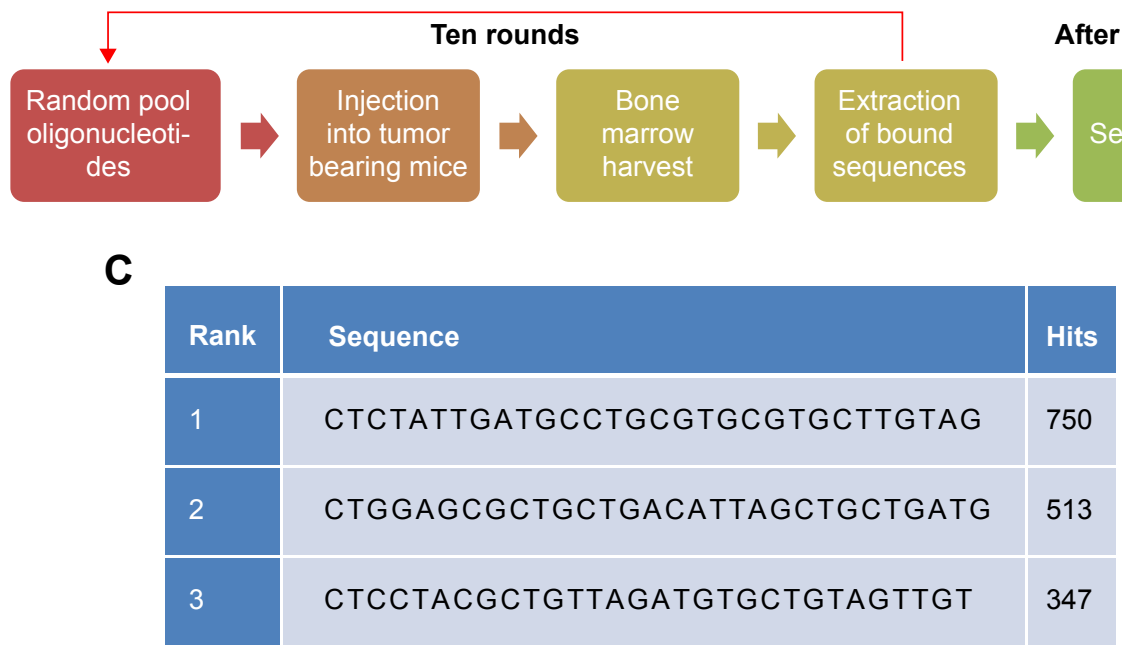

Figure I In vivo SELEX of bone targeting aptamer in nude mice bearing PC3 PB.

Notes: (A) Bioluminescence produced by luciferase in nude mice with PC3 PB (left) and bone marrow tumor sites stained using H\&E (right). (B) In vivo SELEX procedures. (C) The three aptamers with the highest hitting points after SELEX. 
TAG-3') was named by PB (prostate cancer bone metastasis) and selected for further analysis (Figure 1C).

\section{Bone targeting of the PB aptamer in tumor-bearing mice}

Because PB aptamer had the highest hitting frequency among those in the library, it was anticipated to target bone in tumor-bearing mice. Therefore, we analyzed the distribution of Cy5-PB aptamer and Cy5-Control aptamer in different organs after vein injection ex vivo. The PB aptamer exhibited much higher levels of accumulation in bone compared with the control aptamer (Figure 2), consistent with in vivo SELEX outcome. In addition, the bone targeting efficiency of PB aptamer was compared in tumor-bearing and healthy mice. The results showed that the PB aptamer exhibited much higher levels of accumulation in bone of tumor-bearing mice than healthy controls, indicating that the PB aptamer specifically targeted bone in tumor-bearing mice (Figure 2). These properties of bone-specific accumulation in tumor-bearing mice indicated that the PB aptamer had potential as a bone targeting moiety for use in drug delivery.

\section{PB aptamer targets bone marrow endothelial cell}

In bone marrow, the endothelium features in the thin capillary walls, no muscle layer, and sparse basement membrane, which facilitates free exchanges between bone interstitial fluid and the vascular fluids. ${ }^{13}$ Hence, endothelial cell represents a barrier to aptamer entry into the bone marrow and preferred binding to endothelial cell can achieve effective accumulation in the bone. Microscopy analysis revealed that the Cy5-PB aptamer showed an endothelium-associated distribution in bone of tumor mouse, suggesting that endothelial cells may be the target of the PB aptamer (Figure 3A). Immunofluorescence staining of endothelial cell marker CD31 demonstrated that Cy5-PB aptamer colocalized with endothelial cells, not only in bone marrow (Figure 3B) but also within tumor in bone (Figure $3 \mathrm{C}$ ), verifying that bone marrow endothelial cells are the target of PB aptamer.

The binding affinities of the PB and control aptamer for bone marrow endothelial cells from tumor mice were compared in vitro. The results showed that the PB aptamer had a higher binding affinity with endothelial cells than
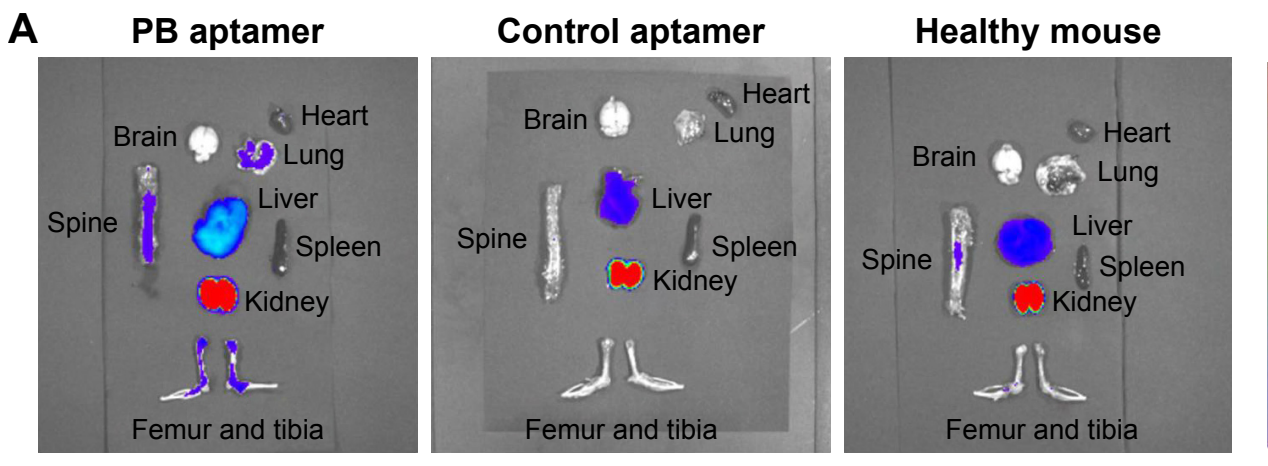

\section{Fluorescence}

$-3.0 \times 10^{9}$

$-2.0 \times 10^{9}$

$-1.0 \times 10^{9}$

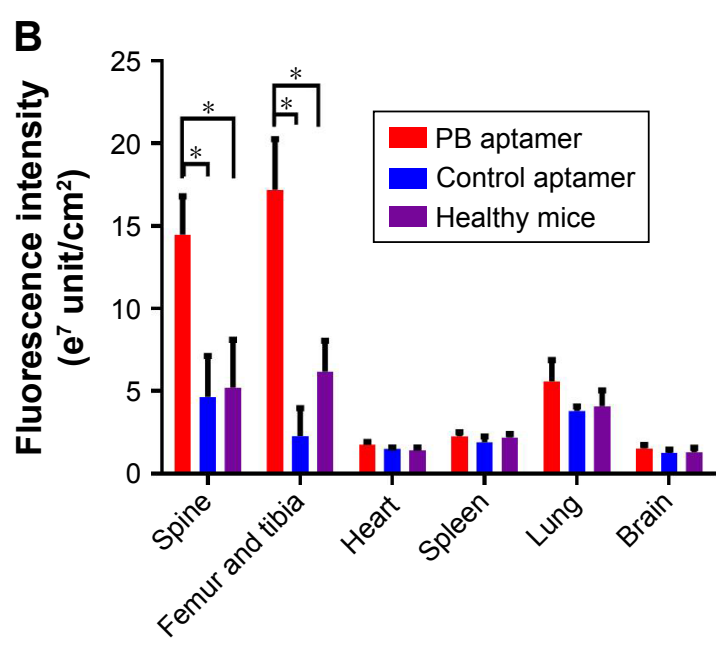

Figure 2 Higher accumulation of the PB aptamer in bone of tumor mouse compared with the control aptamer, more accumulation of the PB aptamer in bone of tumor mice compared with healthy mice $(* P<0.05)$.

Notes: (A) Distribution of the PB aptamer and control aptamer in different organs ex vivo. (B) Fluorescence intensity of aptamer in different organs. 
A
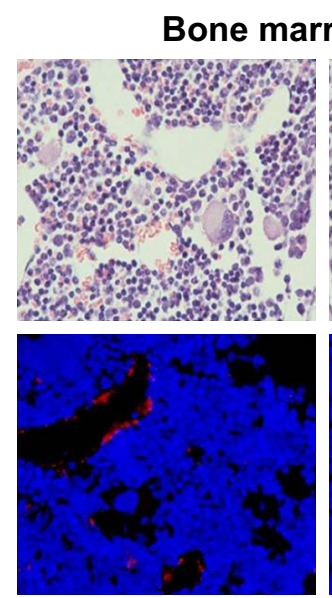

Cy5-PB aptamer/CD31/nuclei

\section{Tumor within bone marrow (40x)}
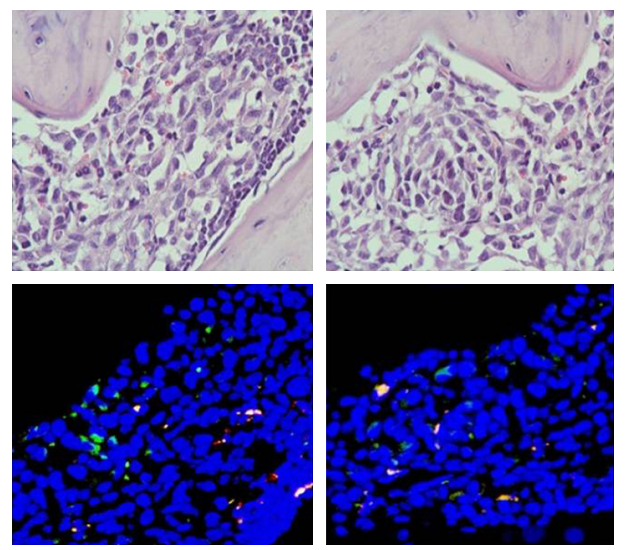

Cy5-PB aptamer/CD31/nuclei
B

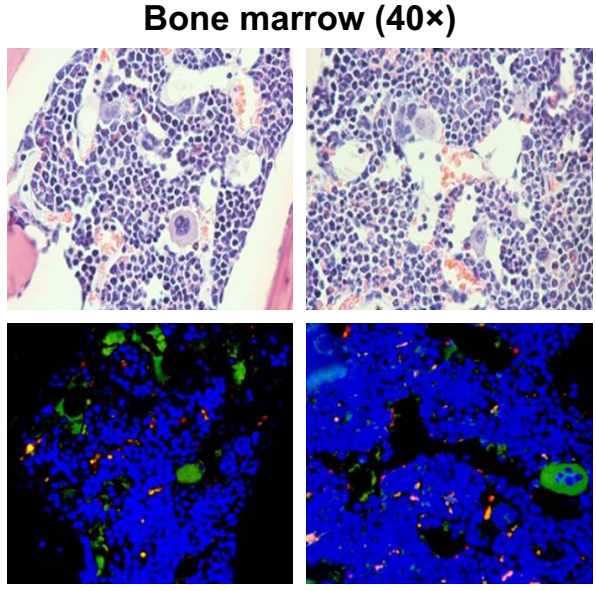

Cy5-PB aptamer/CD31/nuclei

D

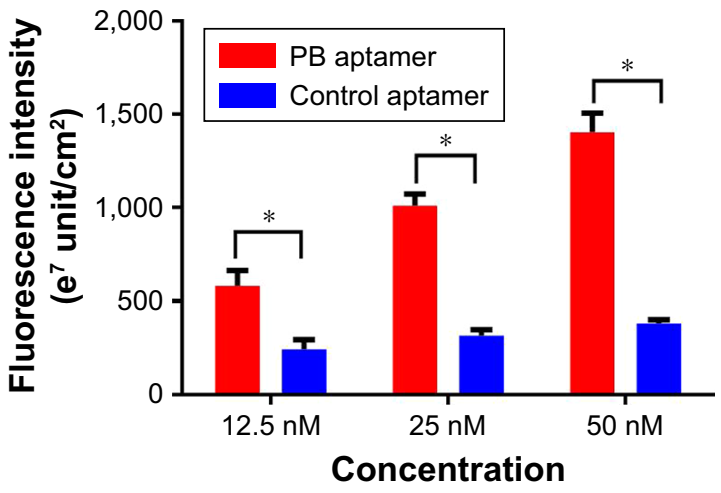

Figure 3 (A) Distribution of the PB aptamer relative to bone marrow vascular endothelium (red: Cy5-PB aptamer; blue: nuclei). (B) Colocalization of PB aptamer with a vascular endothelial cell marker in bone marrow (red: Cy5-PB aptamer; green: CD3I; blue: nuclei). (C) Colocalization of PB aptamer with a vascular endothelial cell marker within tumor in bone marrow (red: Cy5-PB aptamer; green: CD3I; blue: nuclei). (D) Higher binding affinity of PB aptamer to bone marrow endothelial cell compared with control aptamer ex vivo $(* P<0.05)$.

control aptamer (Figure 3D), consistent with specificity of the PB aptamer for bone marrow endothelial cells in tumorbearing mice.

\section{Binding affinity of PB aptamer with HUVEC}

To investigate the bone targeting property of $\mathrm{PB}$ aptamer, HUVEC was utilized to test the interaction between PB aptamer and bone vascular endothelial cell in vivo. The PB aptamer showed specificity for targeting bone in tumorbearing mice; however, the underlying mechanism required further elucidation. We hypothesized that prostate cancer cells from bone could stimulate large amount of PB aptamer affinity molecules on endothelial cell surface as binding ligands. To mimic endothelial cell in vivo, we cultured HUVECs in medium conditioned with bone marrow, or bone marrow containing PC3 prostate cancer, and compared their uptake of the PB aptamer. HUVECs grown in medium conditioned with bone marrow containing PC3 prostate cancer cells exhibited markedly higher Cy5-PB aptamer uptake (Figure 4A). Cytometry analysis further reinforced these findings, as PB aptamer uptake increased by almost three times in HUVECs grown in medium conditioned with bone marrow containing PC3 prostate cancer cells, while remaining unchanged in HUVECs grown in medium conditioned with bone marrow (Figure 4B). These results strongly support the hypothesis that the targeting specificity of PB aptamer for bone marrow endothelial cell is attributable to modulation of the local bone marrow environment by PC3 prostate cancer cells.

The effect of LNCaP or DU145 prostate cancer cell on PB aptamer uptake in HUVEC was also explored. The results 
A

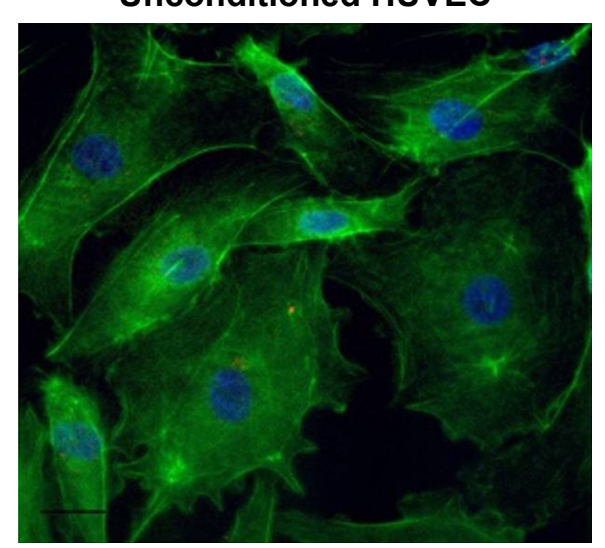

Cy5-PB aptamer/actin/nuclei

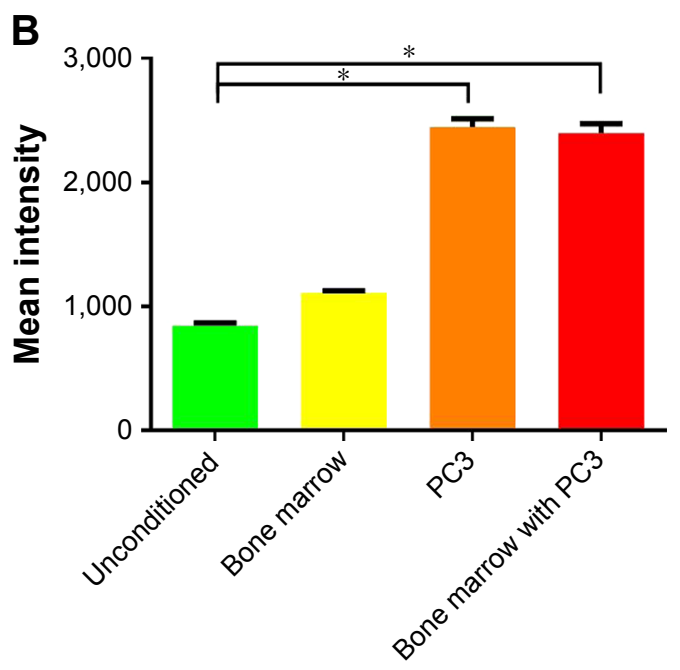

\section{Conditioned HUVEC}

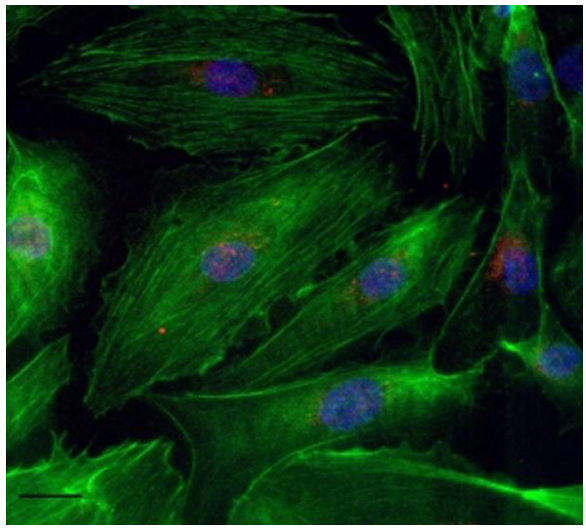

Cy5-PB aptamer/actin/nuclei

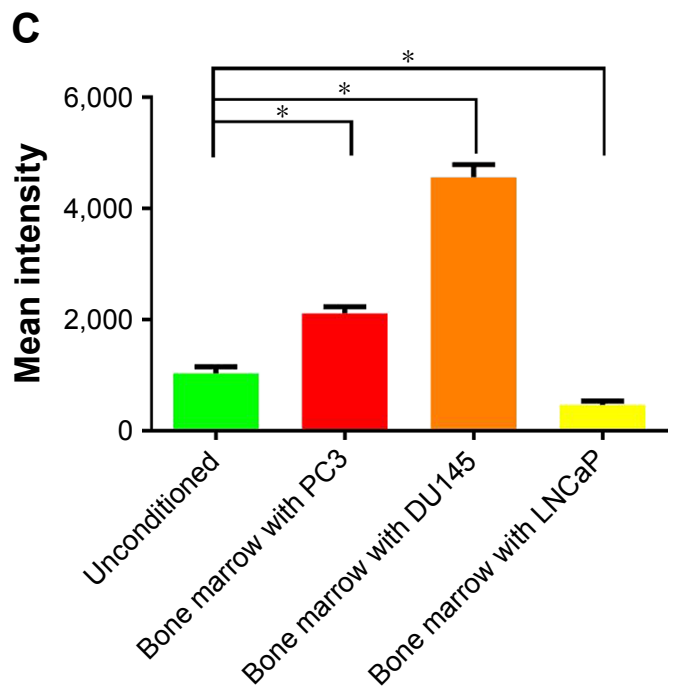

Figure 4 (A) Increased PB aptamer uptake in HUVEC grown in PC3 prostate cancer cell-conditioned medium under fluorescence microscopy (red: Cy5-PB aptamer; green: actin; blue: nuclei). Scale bar: $20 \mu \mathrm{m}$. (B) Increased PB aptamer uptake in HUVECs grown in PC3 prostate cancer cell-conditioned medium ( $<<0.05)$. (C) Increased PB aptamer uptake in HUVECs grown in bone marrow with PC3 or DUI45 prostate cancer cell-conditioned medium, while decreased PB aptamer uptake in HUVECs grown in bone marrow with $\mathrm{LNCaP}$ prostate cancer cell-conditioned medium $(* \mathrm{P}<0.05)$.

Abbreviation: HUVECs, human umbilical vein endothelial cells.

showed that conditioned medium with bone marrow containing LNCaP prostate cancer cells greatly reduced $\mathrm{PB}$ aptamer uptake by HUVECs, while conditioned medium with bone marrow containing DU145 prostate cancer cells stimulated a more significant increase in PB aptamer uptake by HUVECs (Figure 4C). This result suggested that different tumor cells have remarkably diverse effects on bone marrow vascular endothelial cells and that these effects determine aptamer targeting specificity.

\section{PB aptamer cellular internalization pathway and lysosome escape}

Since PB aptamer could be efficiently taken up by PC3 prostate cancer cell-conditioned HUVEC, we investigated the cellular internalization pathway for the PB aptamer.
We hypothesized that the high binding affinity of PB aptamer with HUVEC corresponded to a strong affinity of the aptamer for specific receptors on the surface of HUVEC and that ligand-mediated endocytosis was, therefore, likely to play a major role in the cellular internalization of $\mathrm{PB}$ aptamer. To test this hypothesis, we investigated the uptake of $\mathrm{PB}$ aptamer by HUVECs treated with different cell endocytosis pathway inhibitors. The results showed that cytochalasin D and genistein (both phagocytosis inhibitors) had no effect on PB aptamer uptake by HUVEC, and amiloride (pinocytosis inhibitor) led to an $\sim 30 \%$ decrease in uptake, while chlorpromazine (clathrin-mediated endocytosis inhibitor) and dynasore (dynamin-mediated endocytosis inhibitor), both belonging to ligand-mediated endocytosis inhibitors, inhibited around $70 \%-80 \%$ (Figure $5 \mathrm{~A}$ ). These data indicate that 


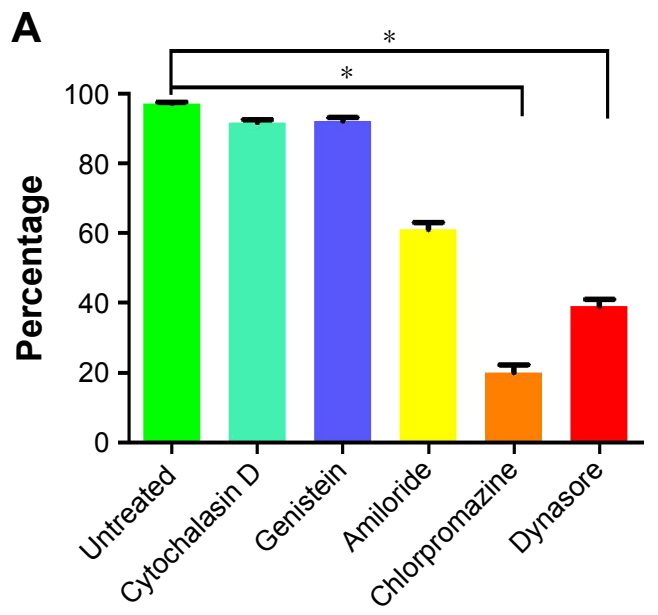

B
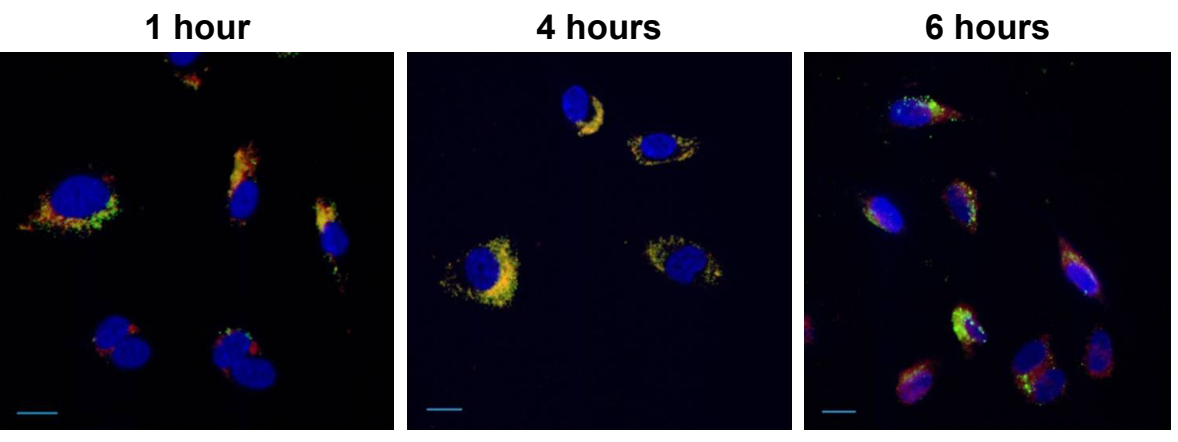

Cy5-PB aptamer/lysosome/nuclei

Figure 5 (A) Decreased PB aptamer uptake by chlorpromazine (clathrin-mediated endocytosis inhibitor) and dynasore (dynamin-mediated endocytosis inhibitor) treated HUVEC $(* P<0.05)$. (B) Escape of the PB aptamer from lysosome 6 hours after HUVEC internalization (red: Cy5-PB aptamer; green: lysosome; blue: nuclei). Scale bar: $20 \mu$ m. Abbreviation: HUVEC, human umbilical vein endothelial cell.

the PB aptamer enters HUVEC mainly via ligand-mediated endocytosis and that the receptors for the PB aptamer are widely expressed on the surface of endothelial cell.

Nanoparticles taken up by endocytosis eventually fused with lysosome in which the highly acidic environment and rich with enzymes promote the degradation of organic nanoparticles. ${ }^{14}$ To track the fate of PB aptamer after cellular internalization, endosomes in HUVEC were stained at several time points after $\mathrm{PB}$ aptamer internalization. The results revealed that PB aptamer escaped lysosome 6 hours after cellular internalization (Figure 5B). This lysosome escape property indicated that PB aptamer could avoid degeneration after vascular endothelial cell internalization, highlighting its potential to function as a protective carrier of conjugated drugs.

\section{Ability of the PB aptamer to target the endothelium and deliver active drugs to bone}

To evaluate whether the PB aptamer could efficiently deliver therapeutics to bone, we prepared Cy5-PB aptamerconjugated gold particles (Figure 6A) and administered them to nude mouse bearing PC3 prostate bone metastasis. Four hours after injection, much higher levels of Cy5-PB-Au particles were observed in bone marrow compared with control Cy5-Au particle (Figure 6B), demonstrating that the PB aptamer could actively deliver drugs to the bone of mice with PC3 prostate bone metastasis. By immunofluorescence staining of endothelium in bone, we detected the colocalization of Cy5-PB-Au particle with bone marrow endothelial cell, confirming the endothelium-targeted accumulation of Cy5-PB-Au aptamer particle consistent with the targeting characteristics of the PB aptamer (Figure 6C).

\section{Discussion}

Nanotechnology is the manipulation of matter on a nanometric scale. Through this technology, it is possible to develop innovative devices with distinct physicochemical properties and enormous therapeutic and diagnostic potential. ${ }^{9}$ As tumor vessels are frequently abnormal, exhibiting increased permeability, nanomedicines with a tendency to enhanced accumulation at the tumor site can improve therapeutic index and reduce side effects. ${ }^{15,16}$ 
A

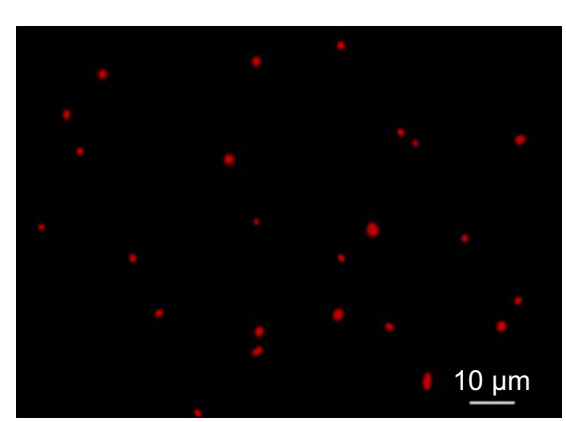

B

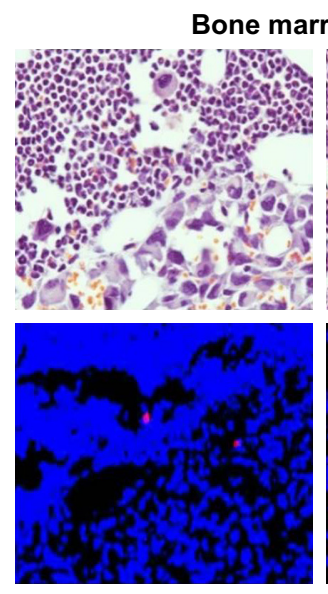

Cy5-Au particle/ nuclei
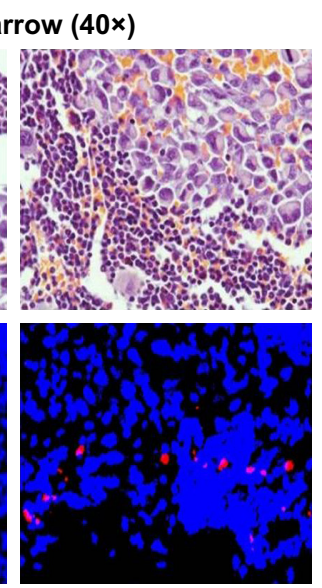

Cy5-PB-Au particle/

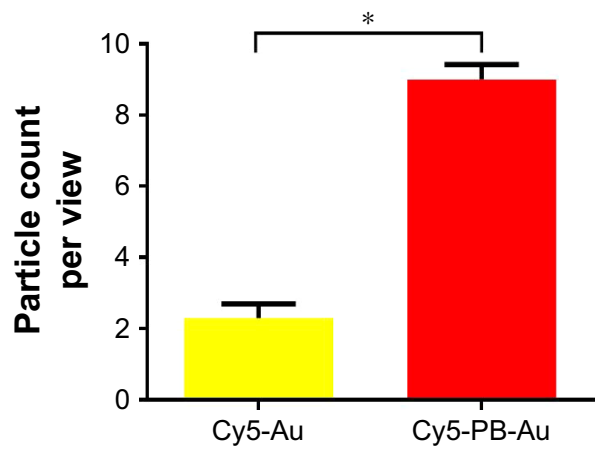

C

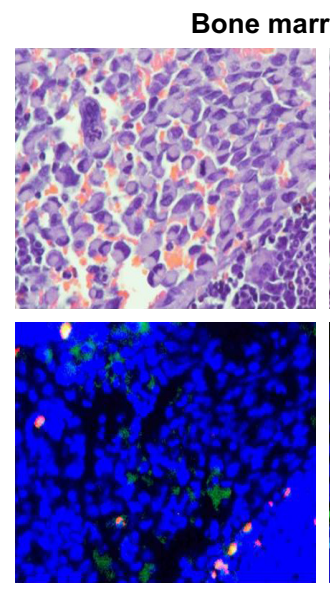

Cy5-PB-Au particle/CD31/nuclei

Figure 6 (A) Cy5-PB-Au particle under fluorescence microscopy. (B) Accumulation of a few Cy5-Au particles within tumor in bone marrow and marked accumulation of Cy5-PB-Au particle within tumor in bone marrow (red: Cy5-Au/Cy5-PB-Au particle; blue: nuclei) $(* P<0.05)$. (C) Colocalization of Cy5-PB-Au particle with a vascular endothelial cell marker within tumor in bone marrow (red: Cy5-PB-Au particle; green: CD3I; blue: nuclei).

Tumor environment is characterized by profound changes in molecular and protein expression profile compared with normal tissue. An appropriate aptamer can specifically bind to unique molecule or cell within the tumor environment and conjugation of such aptamer to drugs can facilitate accumulation of the cargo at a targeted area and achieve targeted drug delivery. Therefore, aptamers function as targeting moieties with important roles in drug delivery. Targeted molecules should be distributed on the surface of target cells, where they represent accessible motifs for their corresponding aptamer.

Our purpose in conducting in vivo SELEX was to obtain aptamers with high binding affinity and specificity for mouse bone with PC3 PB. After ten rounds of in vivo SELEX, the PB aptamer with the highest hitting frequency was attained and analyzed in vitro and in vivo. Compared with control aptamer, the PB aptamer exhibited high levels of accumulation in tumor mouse bone mediated by binding with bone marrow endothelial cell. These conclusions were supported by observations of bone sections under microscopy, as well as high binding affinity of the PB aptamer for mouse tumor-exposed bone marrow endothelial cell ex vivo. Targeting specificity of the PB aptamer was reflected by increased PB aptamer uptake in HUVECs grown in PC3 prostate cancer cell-conditioned medium. In addition, the decreased $\mathrm{PB}$ aptamer uptake in $\mathrm{LNCaP}$ prostate cancer-conditioned HUVEC and increased PB aptamer uptake in DU145 prostate cancer cell-conditioned HUVEC imply that different tumor cells exert huge different effects to reshape local endothelial cell phenotype, thereby determining the targeting specificity of aptamer. We also demonstrated that the PB aptamer underwent ligand-mediated endocytosis into HUVECs, reflecting the abundance of ligands bound by the PB aptamer on the HUVEC surface and consistent with an active targeting strategy. The ability of the PB aptamer to escape from lysosomes indicates that this molecule is a promising choice for application in targeted drug delivery, as it could potentially facilitate its conjugated drugs in avoidance from highly acidic environment and lytic enzymes, thus preserving drugs' bioactivity to achieve desired therapeutic effect. 
The specificity and efficiency of PB aptamer conjugated with gold particles was highlighted by its ability to achieve targeted drug delivery.

Targeting of the tumor microenvironment represents a promising therapeutic approach. In vivo SELEX is a direct and reliable technique for the identification of aptamers that selectively recognize diseased organs or tissues in animal models. ${ }^{17}$ Using selected aptamers, we can develop targeting moiety-equipped nano-based delivery systems with high efficiency and specificity to overcome particularly robust biological barrier, such as bone and brain, and realize targeted drug delivery. The tumor endothelial cell-based active targeting strategy developed in this study can deliver therapeutics to tumor adjacent area to kill cancer cells and has great value in targeting tumor endothelial cells. Targeting tumor endothelial cell directly can contribute to curing cancer by inhibiting angiogenesis, as cancer development is an angiogenesis-dependent process. ${ }^{18-20}$ Inhibition of tumor angiogenesis has been shown as an effective anticancer treatment. ${ }^{21-25}$ Moreover, tumor endothelial cells are unlikely to develop resistance to therapies. ${ }^{26}$ Finally, active aptamer targeting may also improve the therapeutic efficacy of traditional chemotherapy drugs, thereby providing further benefits.

\section{Conclusion}

Tumor cells within bone marrow have a profound influence on local environment, especially on endothelial cells, which provides an opportunity to develop active targeting aptamer for directed drug delivery. In vivo SELEX conducted in this study facilitated the acquisition of an aptamer with high targeting efficiency that can function as a moiety for bonetargeted drug delivery in PB. Aptamer-mediated endothelium targeting could be an alternatively promising strategy for the eradication of cancer growth. Overall, this investigation highlights a potentially bright future for the application of aptamers in the clinic.

\section{Abbreviations}

SELEX, Systematic Evolution of Ligands by Exponential Enrichment; PB, prostate cancer bone metastasis.

\section{Acknowledgment}

This work was supported by a Visiting Scholar Foundation of Key Laboratory of Biorheological Science and Technology (Chongqing University), Ministry of Education (No CQKLBST-2018-005).

\section{Disclosure}

The authors report no conflicts of interest in this work.

\section{References}

1. Siegel RL, Miller KD, Jemal A. Cancer statistics, 2017. CA Cancer J Clin. 2017;67(1):7-30.

2. Bonapace L, Coissieux MM, Wyckoff J, Mertz KD, Varga Z, Junt T, Bentires-Alj M. Cessation of CCL2 inhibition accelerates breast cancer metastasis by promoting angiogenesis. Nature. 2014;515(7525):130-133.

3. Lluch A, Cueva J, Ruiz-Borrego M, Ponce J, Pérez-Fidalgo JA. Zoledronic acid in the treatment of metastatic breast cancer. Anticancer Drugs. 2014;25(1):1-7.

4. Casas A, Llombart A, Martín M. Denosumab for the treatment of bone metastases in advanced breast cancer. Breast. 2013;22(5):585-592.

5. Kang Y, Siegel PM, Shu W, et al. A multigenic program mediating breast cancer metastasis to bone. Cancer Cell. 2003;3(6):537-549.

6. Yin JJ, Selander K, Chirgwin JM, et al. TGF-beta signaling blockade inhibits PTHrP secretion by breast cancer cells and bone metastases development. J Clin Invest. 1999;103(2):197-206.

7. Eckhardt BL, Francis PA, Parker BS, Anderson RL. Strategies for the discovery and development of therapies for metastatic breast cancer. Nat Rev Drug Discov. 2012;11(6):479-497.

8. Hong CA, Nam YS. Functional nanostructures for effective delivery of small interfering RNA therapeutics. Theranostics. 2014;4(12): 1211-1232.

9. Miele E, Spinelli GP, Miele E, Di Fabrizio E, Ferretti E, Tomao S, Gulino A. Nanoparticle-based delivery of small interfering RNA: challenges for cancer therapy. Int J Nanomedicine. 2012;7:3637-3657.

10. Dang L, Liu J, Li F, et al. Targeted delivery systems for molecular therapy in skeletal disorders. Int J Mol Sci. 2016;17(3):428-443.

11. Sun $\mathrm{H}, \mathrm{Zu}$ Y. Aptamers and their applications in nanomedicine. Small. 2015;11(20):2352-2364.

12. Liang $\mathrm{C}, \mathrm{Guo} \mathrm{B}, \mathrm{Wu} \mathrm{H}$, et al. Aptamer-functionalized lipid nanoparticles targeting osteoblasts as a novel RNA interference-based bone anabolic strategy. Nat Med. 2015;21(3):288-294.

13. Cowin SC, Cardoso L. Blood and interstitial flow in the hierarchical pore space architecture of bone tissue. J Biomech. 2015;48(5):842-854.

14. Blanco E, Shen H, Ferrari M. Principles of nanoparticle design for overcoming biological barriers to drug delivery. Nat Biotechnol. 2015;33(9): 941-951.

15. Carmeliet P. Angiogenesis in life, disease and medicine. Nature. 2005;438(7070):932-936.

16. Fukumura D, Jain RK. Tumor microvasculature and microenvironment: targets for anti-angiogenesis and normalization. Microvasc Res. 2007;74(2-3):72-84

17. Mi J, Ray P, Liu J, et al. In vivo selection against human colorectal cancer xenografts identifies an aptamer that targets RNA helicase protein DHX9. Mol Ther Nucleic Acids. 2016;5:e315.

18. Folkman J. Toward an understanding of angiogenesis: search and discovery. Perspect Biol Med. 1985;29(1):10-36.

19. Kerbel RS. Inhibition of tumor angiogenesis as a strategy to circumvent acquired resistance to anti-cancer therapeutic agents. Bioessays. 1991;13(1):31-36.

20. Folkman J. Angiogenesis in cancer, vascular, rheumatoid and other disease. Nat Med. 1995;1(1):27-30.

21. Ingber D, Fujita T, Kishimoto S, et al. Synthetic analogues of fumagillin that inhibit angiogenesis and suppress tumour growth. Nature. 1990;348(6301):555-557.

22. Kim KJ, Li B, Winer J, Armanini M, Gillett N, Phillips HS, Ferrara N. Inhibition of vascular endothelial growth factor-induced angiogenesis suppresses tumour growth in vivo. Nature. 1993;362(6423):841-844.

23. O'Reilly MS, Holmgren L, Shing Y, et al. Angiostatin: a novel angiogenesis inhibitor that mediates the suppression of metastases by a Lewis lung carcinoma. Cell. 1994;79(2):315-328. 
24. Brekken RA, Overholser JP, Stastny VA, Waltenberger J, Minna JD, Thorpe PE. Selective inhibition of vascular endothelial growth factor (VEGF) receptor 2 (KDR/Flk-1) activity by a monoclonal anti-VEGF antibody blocks tumor growth in mice. Cancer Res. 2000;60(18): $5117-5124$.
25. Ishida T, Kundu RK, Yang E, Hirata K, Ho YD, Quertermous T. Targeted disruption of endothelial cell-selective adhesion molecule inhibits angiogenic processes in vitro and in vivo. J Biol Chem. 2003;278(36):34598-34604.

26. Aird WC. Endothelial cell heterogeneity. Cold Spring Harb Perspect Med. 2012;2(1):a006429.

International Journal of Nanomedicine

\section{Publish your work in this journal}

The International Journal of Nanomedicine is an international, peerreviewed journal focusing on the application of nanotechnology in diagnostics, therapeutics, and drug delivery systems throughou the biomedical field. This journal is indexed on PubMed Central, MedLine, CAS, SciSearch $\AA$, Current Contents ${ }^{\circledR} /$ Clinical Medicine,
Journal Citation Reports/Science Edition, EMBase, Scopus and the Elsevier Bibliographic databases. The manuscript management system is completely online and includes a very quick and fair peer-review system, which is all easy to use. Visit http://www.dovepress.com/ testimonials.php to read real quotes from published authors.

Submit your manuscript here: http://www.dovepress.com/international-journal-of-nanomedicine-journal 